\title{
Medida da área do canal vertebral lombar em diferentes faixas etárias
}

\author{
Lumbar spinal canal area in different age groups \\ Medida de la superficie del canal vertebral lumbar en los diferentes \\ grupos de edad
}

Jair Ortiz'

Alex Veneziano Oliveira Junior ${ }^{2}$

José Luis Amim Zabeu ${ }^{3}$

\section{RESUMO}

Objetivo: medir as dimensões do canal vertebral lombar por meio de tomografia computadorizada, de modo a comparar os valores das diferentes faixas etárias e determinação da possível diferença entre elas. Métodos: a área do canal vertebral lombar foi medida em 78 indivíduos, divididos em oito grupos etários, com dados obtidos de exames de tomografia computadorizada do abdômen. As medidas foram realizadas nos cortes axiais nas vértebras L1, L3 e L5 ao nível da região trans-pedicular. Utilizado o método estatístico de ANOVA (análise de variância) com análise de diferentes "p-value" para cada grupo estudado. Resultados: as medidas para L1 produziram um "p-value" de 0,586 para a faixa etária e um "p-value" de 0,003 para o sexo. Portanto, não há diferença entre as faixas etárias e há diferença entre os sexos, sendo maior no sexo masculino. Para L3, o "p-value" da faixa etária foi de 0,258 e para

\section{ABSTRACT}

Objective: to measure the lumbar spinal canal area, using computerized tomography, compare different age groups and determine if there are differences between those. Methods: the lumbar spinal canal area was measured in 78 individuals, divided in eight age groups, with data obtained from computerized tomography scans of the abdomen. The measurements were made at L1, L3 and L5 levels at the trans- pedicular section. Results: the values for $L 1$ produced a $p$-value of 0,586 for the age group and a $p$-value of 0,003 for sex. Therefore, we can say that there is no evidence of differences among age rage groups, but shows evidence of difference for sex being larger in males. For L3, the p-value of age rage was 0,258 , and the value for sex was 0,062. For $L 5$, the p-value for the age range was 0279, and the value for sex was 0,003. Conclusion: there is no difference of spinal canal area between the
\end{abstract}

\section{RESUMEN}

Objetivo: medir el área del canal vertebral lumbar, utilizando la tomografia computarizada, comparándolo entre los distintos grupos de edad y determinar si existen diferencias entre ellos. Métodos: el área del canal vertebral lumbar se midió en 78 temas, divididos en 8 grupos, con los datos obtenidos a partir de exploraciones de tomografía computarizada del a domen. Las mediciones se hicieron en las vértebras L1, L3 y L5 en la región transpedicular. Resultados: medidas para L1 producido un p-valor de 0,586 para el grupo de edad y un p-valor de 0,003 para el sexo, por tanto, no hay diferencia entre los grupos de edad y hay diferencias entre los sexos, siendo mayor en los hombres; para L3, el "pvalue" del rango de edad fue 0,258 y para el sexo de 0,062; en el caso de la L5, el "p-value" para el rango de edad fue 0,279 y el sexo de 0,003. Conclusión: no hay diferencia en el ámbito de la canal vertebral lumbar

\footnotetext{
Trabalho realizado no Departamento de Ortopedia e Traumatologia do Hospital e Maternidade Celso Pierro da Pontifícia Universidade Católica de Campinas PUCCAMP, Campinas (SP), Brasil.

'Médico Assistente do Grupo de Coluna do Departamento de Ortopedia e Traumatologia do Hospital e Maternidade Celso Pierro da Pontifícia Universidade Católica de Campinas - PUCCAMP- Campinas (SP), Brasil.

${ }^{2}$ Residente do $3^{\circ}$ ano do Programa de Residência Médica em Ortopedia e Traumatologia do Hospital e Maternidade Celso Pierro da Pontifícia Universidade Católica de Campinas - PUCCAMP - Campinas (SP), Brasil.

${ }^{3}$ Chefe do Departamento de Ortopedia e Traumatologia do Hospital e Maternidade Celso Pierro. PUCCAMP - Campinas (SP), Brasil.
} 
o sexo de 0,062 ; no caso de L5, o "pvalue" para a faixa etária foi de 0,279 e para o sexo de 0,003. Conclusão: não foi observada diferença estatística da área do canal vertebral lombar nos níveis L1, L3 e L5 nas diferentes faixas etárias. No entanto o diâmetro no sexo masculino foi maior com diferença estatística.

DESCRITORES: Canal vertebral/crescimento \& desenvolvimento; Tomografia computadorizada por raios $\mathrm{X}$; Grupos etários age groups, but there is difference for the sex being larger in males. entre los grupos de edad analizados, pero hay diferencias entre los sexos, siendo mayor en los hombres.
KEYWORDS: Spinal canal/growth \& development; Tomography, X-ray computed; Age group

\author{
DESCRIPTORES: Conducto \\ vertebral /crecimiento \& \\ desarrollo; Tomografia \\ computarizada por rayos $\mathrm{X}$; \\ Grupos por edad
}

\section{INTRODUÇÃO}

Cada segmento da coluna vertebral (cervical, torácica e lombar) possui características anatômicas próprias, conforme sua origem embrionária e exigência mecânica. $\mathrm{O}$ diâmetro e a forma do canal vertebral variam ao longo dos diferentes níveis da coluna vertebral. A vértebra lombar é peculiar, pois não tem articulação com as costelas, possui extenso processo espinhoso, assim como extensos pedículos e lâminas e a forma do canal vertebral lombar, varia desde uma forma oval até triangular1. O canal vertebral compreende o espaço delimitado pelo corpo vertebral à frente, os pedículos e facetas articulares lateralmente e as lâminas na região posterior. O canal vertebral lombar contém o cone medular e a cauda equina. Assim como os vasos epidurais e uma quantidade variada de gordura em torno da dura-máter resultando em um mínimo espaço livre entre o canal e o seu conteúdo. O tamanho e a forma normal do canal medular são importantes, pois permitem o movimento do seu conteúdo sem tensão ou pressão ${ }^{1}$.

A coluna fetal humana se torna madura na infância e o diâmetro interpedicular, no nível de L1 a L4, no nascimento, é aproximadamente $70 \%$ do diâmetro do adulto. A morfologia do canal lombar alcança o tamanho do adulto com um ano de idade. Qualquer insulto ao desenvolvimento, na vida embrionária ou na infância, pode influenciar no desenvolvimento do canal vertebra ${ }^{12}$. Aos quatro anos de idade, o diâmetro sagital do canal vertebral e a distância interpedicular apresenta $87 \%$ do diâmetro do adulto ${ }^{3}$.

Existem fatores que se relacionam com o desenvolvimento do canal vertebral, como: peso placentário, baixo peso ao nascimento, baixo nível sócio-econômico ${ }^{1}$.

A morfologia do canal vertebral foi avaliada na população coreana, para determinar os valores normais na coluna lombar para esta população e, então, verificar se há alguma diferença racial nos valores do canal vertebral lombar, e fornecer subsídio para o diagnóstico de estenose espinhal em asiáticos. Alguns estudos radiológicos e anatômicos têm sido feitos, considerando-se o tamanho do canal vertebral lombar de brancos e negros nos países ocidentais e africanos ${ }^{4}$. A correlação da dimensão vertebral com parâmetros somatométricos foi realizada por meio da tomografia computadorizada. Os resultados mostraram que há uma correlação significante da estatura, peso e idade com os vários índices vertebrais (dimensões do canal vertebral lombar)

A definição de valores normais do canal vertebral lombar, nas diferentes faixas etárias, é necessária porque talvez possa explicar as doenças dolorosas da coluna lombar como, por exemplo, a estenose espinhal.

Em indivíduos com idade entre 22 a 74 anos, processos degenerativos nos segmentos vertebrais lombar podem alterar o tamanho e a forma do canal vertebral. A intensidade e o grau desta degeneração variam entre diferentes faixas etárias6. Medidas do tamanho do canal vertebral podem ser determinadas por exames radiográficos convencionais, porém a melhor avaliação é realizada por meio da ressonância magnética ou tomografia computadorizada ${ }^{7}$.

O presente trabalho tem o objetivo de medir a área do canal vertebral da coluna lombar em diferentes faixas etárias por meio da tomografia computadorizada.

\section{MÉTODOS}

A área do canal vertebral lombar foi medida em 78 indivíduos 37 do sexo feminino, 40 do sexo masculino e um não determinado. A média de idade foi de 40,72 $( \pm 2,56)$ anos. $\mathrm{O}$ indivíduos foram divididos em oito grupos etários com intervalos de dez anos (de 1 a 10, de 11 a 20, de 21 a 30, de 31 a 40 , de 41 a 50 , de 51 a 60 , de 61 a 70 e de 71 a 80 anos. Os dados foram obtidos dos exames de tomografia computadorizada do abdômen realizados por doenças não vertebrais, na incidência axial. A medida foi feita nas vértebras L1, L3 e L5 ao nível da região transpedicular. Todas as medidas foram realizadas por um dos autores, em conjunto com o mesmo radiologista. Não foram considerados raça e dados antropométricos. A tabela 1 define as variáveis observadas no estudo. Como primeira etapa, efetuou-se uma visão panorâmica da coluna lombar lateral, para estabelecer o nível de corte, que foi no nível transpedicular da primeira, terceira e quinta vértebra lombar, onde foi avaliada a área do canal vertebral (Figura 1). 
TABELA 1 : Descrição das variáveis estudadas

\begin{tabular}{lll}
\hline Variável & Tipo & Escalas/Valores \\
\hline Idade & Discreta & Anos \\
\hline Sexo & Categorizada & M ou F \\
\hline L1 & Contínua & Maior que zero \\
L3 & Contínua & Maior que zero \\
L5 & Contínua & Maior que zero \\
\hline Faixa & Categorizada & Classes descritas acima
\end{tabular}

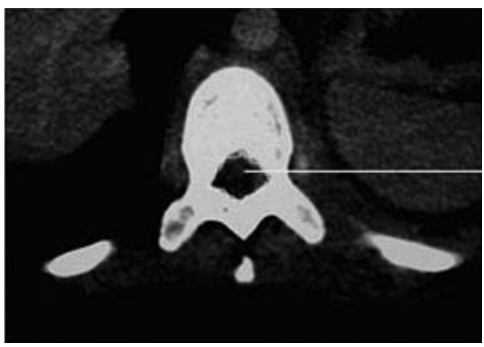

Área do canal vertebral

Figura 1

Vertebra lombar

Fonte: Arquivo do Departamento de Radiologia

\section{RESULTADOS}

Os resultados referentes ao comportamento das variáveis de interesse: L1, L3 e L5 com a distribuição dos pacientes quanto ao sexo dentro de cada faixa etária são demonstrados na tabela 2 .

Nota-se que as três últimas faixas não estão balanceadas quanto ao número de pacientes por sexo. As colunas L1, L3 e L5 apresentam as médias das variáveis, em cada faixa, com o respectivo erro padrão. Observa-se que em L1, os valores médios entre as faixas são próximos (considerando-se o erro padrão), com exceção apenas a primeira. O mesmo ocorre em L3, mas agora são três faixas que parecem não concordar, quinta, sexta e oitava. Quanto a L5, os resultados apresentam maior variação.

A figura 2 mostra como intervalos para a média, em cada faixa, se comportariam. Isso corrobora o que é visto na Ta- bela 2: enquanto as médias em L1 estão semelhantes, em L5 encontram-se muito variadas. Devido ao pouco número da amostra em cada faixa etária observou-se uma variaçãocáo nos resultados. Os resultados das diferenças entre as médias das faixas mostraram-se prejudicados.

A figura 3 apresenta gráficos de caixas para as variáveis estudadas. Cada caixa representa os valores observados para uma faixa etária. A linha central indica o valor da mediana (valor central entre os observados), os limites da caixa indicam o primeiro quartil (inferior; valor que deixa $25 \%$ dos valores observados abaixo e $75 \%$ acima) e o terceiro quartil (superior; valor que deixa $25 \%$ dos valores observados acima e $75 \%$ abaixo). Sendo assim, dentro da caixa temos representado $50 \%$ da amostra, sendo $25 \%$ abaixo da mediana e acima do primeiro quartil, e $25 \%$ acima da mediana e abaixo do terceiro quartil. As hastes se estendem até o valor máximo (haste superior) ou mínimo (haste inferior). Caixas muito longas podem sugerir maior variação nos dados. Caixas com a mediana deslocada do centro podem sugerir assimetria nos dados. Os asteriscos são valores considerados discrepantes entre os demais. O objetivo é determinar a existência ou não de diferenças significativas entre as áreas através das faixas etárias. A análise é feita em três etapas, uma para cada variável. As variáveis atendem as suposições necessárias para utilização de um modelo de ANOVA (análise de variância $)^{8}$. Esta técnica permite determinar se a variação observada pode ser explicada por fatores específicos dos pacientes, como faixa etária e sexo. Sendo assim, foram construídos três modelos, um para cada variável (L1, L3 e L5), considerando as faixas etárias e o sexo. Segundo o resultado dos testes, rejeitamos a hipótese de que um fator não é significativo quando o $p$-value for menor que o valor de corte previamente determinado (no caso, foi usado 5\%). O modelo para L1 produziu um p-value de 0,586 para a faixa etária. Sendo assim, pode-se dizer que não existem evidências de diferenças entre as faixas etárias. Já o sexo apresentou $p$-value de 0,003 , ou seja, com evidências de diferenças. Para L3, o $p$-value de faixa etária foi de 0,258 , e do sexo de 0,062 . No caso de L5, o $p$-value para a faixa etária foi de 0,279 , e para o sexo de 0,003 .

TABELA 2 - Distribuição das variáveis

\begin{tabular}{lccccccc}
\hline & \multicolumn{7}{c}{ Número de pacientes } \\
\cline { 2 - 6 } Faixa & Total & F & M & $?$ & L1 & L3 & L5 \\
\hline 01 a 10 & 10 & 4 & 5 & 1 & $247,5( \pm 24,9)$ & $249,0( \pm 26,5)$ & $264,6( \pm 26,0)$ \\
11 a 20 & 10 & 4 & 6 & 0 & $309,7( \pm 25,5)$ & $255,9( \pm 20,0)$ & $307,2( \pm 24,8)$ \\
21 a 30 & 9 & 4 & 5 & 0 & $307,2( \pm 20,7)$ & $260,3( \pm 19,0)$ & $299,0( \pm 21,6)$ \\
31 a 40 & 10 & 4 & 6 & 0 & $297,7( \pm 15,7)$ & $262,4( \pm 14,6)$ & $338,0( \pm 13,1)$ \\
41 a 50 & 9 & 4 & 5 & 0 & $286,7( \pm 21,6)$ & $298,2( \pm 22,5)$ & $351,7( \pm 39,3)$ \\
51 a 60 & 10 & 8 & 2 & 0 & $294,1( \pm 23,8)$ & $301,2( \pm 40,7)$ & $327,5( \pm 41,9)$ \\
61 a 70 & 10 & 6 & 4 & 0 & $293,2( \pm 19,2)$ & $281,0( \pm 16,7)$ & $298,5( \pm 17,5)$ \\
71 a 80 & 10 & 3 & 7 & 0 & $299,0( \pm 16,1)$ & $318,1( \pm 15,2)$ & $336,8( \pm 21,9)$ \\
\hline
\end{tabular}

Fonte: Arquivo de tomografia computadorizada do Departamento de Radiologia Medida das áreas avaliadas em milímetros quadrados 
L1

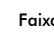

Faixa

11 a 20

21 a 30

31 a 40

41 a 50

51 a 60

61 a 70

71 a 80
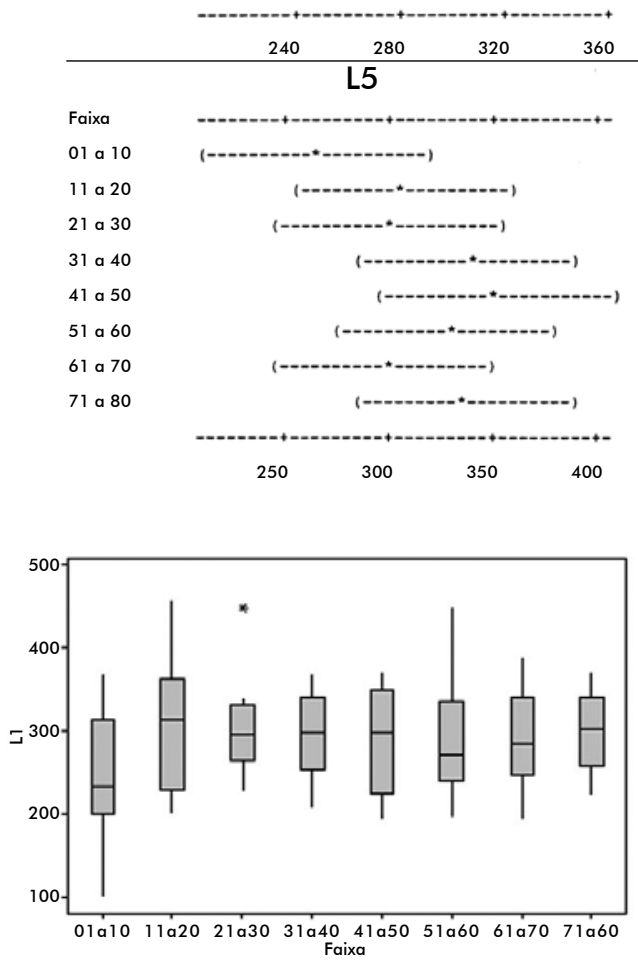

L3

Faixa

01 a 10

11 a 20

21 a 30

31 a 40

41 a 50

51 a 60

61 a 70

71 a 80

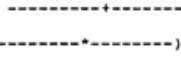

(---------*--------)

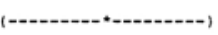

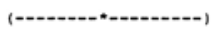

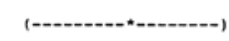

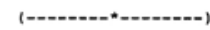

(----------*--------)

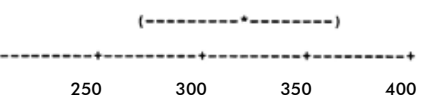

Figura 2

Comportamento médio por faixa etária

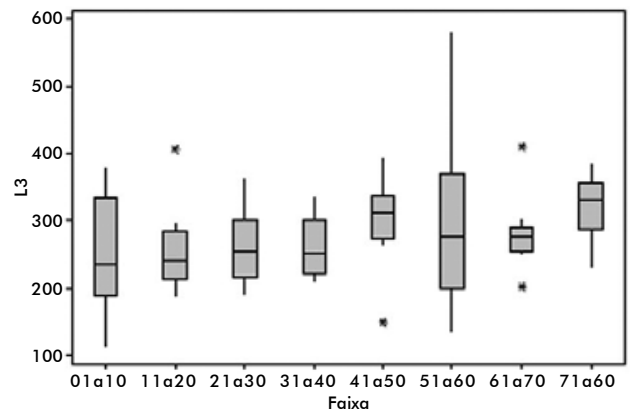

Figura 3

Gráfico de caixas

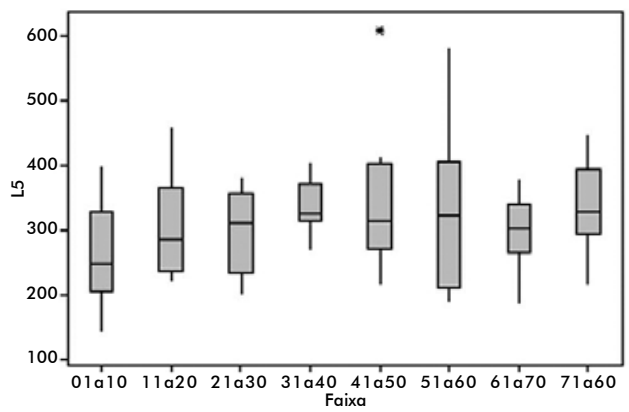

\section{DISCUSSÃO}

O objetivo deste estudo foi comparar o valor da área do canal vertebral lombar em sete diferentes faixas etárias, por meio da tomografia computadorizada. A área do canal vertebral lombar já está totalmente desenvolvida aos quatro anos de idade ${ }^{3}$, porém, nós não encontramos na literatura algo que comparasse, entre diferentes faixas etárias, a medida da área do canal vertebral lombar.

As dimensões da vértebra lombar são estabelecidas com o auxílio dos exames de imagem, tais quais, ressonância magnética e tomografia computadorizada. No pas- sado, usava-se o exame radiográfico convencional, o qual apresentava um problema metodológico para medição: a magnificação das imagens ${ }^{7}$. Este estudo estabelece os valores por meio de técnica digital.

Grande parte do crescimento do canal vertebral lombar acontece na infância, portanto, qualquer atraso do desenvolvimento da criança nesta fase, traduz-se na formação de um canal vertebral com uma área menor ${ }^{9}$, sendo que $87 \%$ do tamanho do canal vertebral já está estabelecido aos quatro anos de idade ${ }^{3}$. Apesar de não termos discrimi- 
nado a idade por unidade e sim por década, concluímos que o tamanho do canal na primeira década manteve-se constante por todas as outras décadas.

Há correlação de parâmetros somatométricos, peso, altura e idade, com a dimensão do canal vertebral lombar ${ }^{5}$. Os nossos dados foram obtidos de um arquivo digital de tomografias computadorizadas, no qual tínhamos acesso apenas à idade e o sexo do paciente. A análise de somente estas duas variáveis foi suficiente para concluirmos que a área do canal vertebral não varia significantemente a partir dos valores da primeira década de vida.

Um estudo determinou, pelas radiografias convencionais, o diâmetro transverso do canal vertebral lombar, comparando-o entre os sexos, sendo no masculino maior que no sexo feminino ${ }^{1}$, o que correspondeu aos nossos achados. As dimensões anatômicas do canal vertebral lombar foram medidas em pacientes asiáticos para definir diferenças entre as diversas raças. Também foram feitos estudos radiológicos e anatômicos que compararam o tamanho do canal vertebral lombar entre brancos e negros. O diâmetro sagital do canal nos coreanos é menor que nos brancos e negros, mas, em relação ao diâmetro transverso do canal vertebral lombar, não há diferenças entre estas raças4. Neste estudo não incluímos a raça dos pacientes.

A aplicação prática dos nossos achados nos leva a entender melhor a doença da estenose do canal lombar, concluindo que a estenose espinal já está presente desde a primeira infância e pode estar associada a insultos nesta faixa etária da vida, ao passo que a sintomatologia passa a ser precipitada pelas alterações degenerativas. Procedimentos cirúrgicos que envolvam a coluna vertebral e que penetram pelo canal, como a aramagem sublaminar, levam a risco de dano neurológico, independente da faixa etária.

\section{CONCLUSÃO}

A área do canal vertebral não tem diferença significativa nas diferentes faixas etárias, mas é maior no sexo masculino.

\section{Agradecimento}

Ao Professor Doutor Gabriel Coelho Gonçalves de Abreu, pesquisador e bioestatístico que realizou a análise estatística deste estudo.

\section{REFERÊNCIAS}

1. Tacar O, Demirant A, Nas K, Altindag O. Morphology of the lumbar spinal canal in normal adults Turks. Yonsei Med J. 2003; 44(4):679-85.

2. Wallny T, Schild R L, Fimmers R, Hansmann M E. Three-dimensional sonographic evaluation of the fetal lumbar spinal canal. J Anat. 2002; 200(5): 439-43.

3. Porter R W, Pavitt D. The vertebral canal: I. Nutrition and development, an archaelogical study. Spine. 1987;12 (9):901-6.

4. Lee H M, Kim N H, Kim H J, Chung I H. Morphometric study of the lumbar spinal canal in the Korean population. Spine. 1995; 20(15): 1679-84.

5. Karantanas A H, Zibis A H, Papaliaga M, Georgiou E, Rousogiannis S. Dimensions of the lumbar spinal canal: variations and correlations with somatometric parameters using CT. Eur Radiol. 1998; 8(9):1581-5.
6. Borisevich A I, Eremeishvili A V. Lumbar portion of the human spinal canal at different age periods. Arkh Anat Gistol Embriol. 1979; 77(11): 12-21.

7. Knirsch W, Kurtz C, Häffner N, Langer M, Kececioglu D. Normal values of the sagittal diameter of the lumbar spine (vertebral body and dural sac) in children measured by MRI. Ped Radiol. 2005; 35(4): 419-24.

8. Johnson RA, Wichern DW. Applied Multivariate Statistical Analysis. 4th ed. New York: Prentice-Hall, Inc.; 1998.

9. Papp T, Porter R W, Aspeden R M. The growth of the lumbar vertebral canal. Spine. 1994; 19(24): 2770-3.

Correspondência
Jair Ortiz
Av. John Boyd Dunlop, s/nº
Jardim Ipaussurama
CEP 13060-904
Campinas, SP, Brasil
Tel.:+ 551933438575
E-mail: driairortiz@yahoo.com.br

